\title{
Effects of Internet Sales Promotion on a Differential Advertising Model
}

\author{
Hui Jiang and Junhai Ma \\ College of Management and Economics, Tianjin University, Tianjin 300072, China \\ Correspondence should be addressed to Junhai Ma; mjhtju@aliyun.com
}

Received 23 November 2017; Accepted 12 March 2018; Published 6 May 2018

Academic Editor: Mustafa R. S. Kulenovic

Copyright ( 2018 Hui Jiang and Junhai Ma. This is an open access article distributed under the Creative Commons Attribution License, which permits unrestricted use, distribution, and reproduction in any medium, provided the original work is properly cited.

\begin{abstract}
Advertising and sales promotion are two important specific marketing communications tools. In this paper, Internet sales promotion is introduced into a differential advertising model and investigated quantitatively. The conditions for the existence and stability of periodic solutions are obtained. Flip bifurcation of periodic solution is investigated analytically. The results show that the sales promotion parameter can modify the stability of the differential advertising model and lead to chaos through flip bifurcation, the sales level will eventually be no less than a given value by adjusting the value of the sales promotion parameter, and the optimal sales promotion strategy can lead to maximum profit. Numerical results for periodic solutions, bifurcation diagrams, and the effects of sales promotion strategies, which are illustrated with an example, are in good agreement with the theoretical analysis. These results have certain significant theoretical and practical value in related markets.
\end{abstract}

\section{Introduction}

Advertising is an audio or visual form of marketing communication that employs an openly sponsored, nonpersonal message to promote or sell a product, service, or idea. The purpose of advertising is to convince customers that a company's services or products are the best, point out and create a need for products or services, announce new products and programs, draw customers to the business, and hold existing customers. There has been a growing interest in the problem of determining profit maximizing advertising strategies by both practitioners and researchers [1-4].

Many discrete and differential game models are constructed to investigate advertising in recent years. A twodimensional map is proposed to describe a market where there are two quantity-setting firms producing homogeneous goods [5]. A discrete market share attraction model, where agents are boundedly rational, is introduced to investigate multistability and path dependence [6] (in this model, brand managers are assumed to be boundedly rational and over time adapt their marketing efforts for their brand in correspondence to the marginal profits of the previous period). Compared to discrete models [7] (by the theory of nonlinear dynamics, the authors embed multiproduct structure in output duopolymixed dynamical game model with partial privatization and cross-ownership in which a private firm and a public firm coexist); [8] (dynamic system concepts are integrated into this game model for understanding the evolution of the strategic decisions over multiple time periods), differential game models are more suitable for the mathematical simulation of evolutionary processes in advertising. Recent years have seen many results about differential game models (see, e.g., [9]). Su et al. [10] use the dynamic evolutionary game model and the multiagent modeling simulation to discuss the APQSIS agents equilibrium strategies and the effects of their interactive behaviors on the APQSIS evolutionary stability with asymmetric information. Feinberg [11] assumes that the market share evolves according to a differential game model with an S-shaped function, provides a rational as to when the nonconstant advertising policy is optimal, and uses the classic phase-plane analysis to deal with the unspecified nonlinearity in the response function (casting the dynamic between advertising and sales in a common format (an autonomous, first-order relationship), extensions are explored along three dimensions: an S-shaped response function, the value of the discount rate, and the possibility of 
diffusion like response). A differential game model is used to obtain firms marketing communications decisions in markets with uncertainty and competition [12]. Krishnamoorthy et al. [13] consider a duopoly differential game model with joint advertising and pricing decisions and obtain the closedform Nash equilibria for both the game with symmetric competitors and that with asymmetric competitors by using the linear and isoelastic demand function forms (differential game theory is used to analyze two different demand specifications-linear demand and isoelastic demand-for symmetric and asymmetric competitors). Despite the useful results gained from differential game models in advertising, the complex dynamical behaviors such as periodic solution and bifurcation are rarely investigated in advertising models. Su et al. [14] establish the trilateral game payoff matrix, build up the replicator dynamic equations, and discuss possible evolutionary stable states.

Sales promotion [15] (this study proposes that the responses of more and less deal-prone consumers to price discounts and premiums depend on the promotional benefit level; low deal-prone consumers are concerned with obtaining price discounts), including things like contests and games, sweepstakes, product giveaways, samples coupons, loyalty programs, and discounts, is another way to advertise. The ultimate goal of sales promotions is to stimulate potential customers to action and create an immediate boost in sales volume. Previous studies mainly use the methods of dataanalysis and quality analysis to discuss the role of sales promotion. For example, the effects of sales promotions on brand preference are discussed and it is found that sales promotions do not affect postpromotion brand preference by using meta-analysis [16]. Promotion cost is considered in a model which shows that keeping the fractions of promotion cost sharing within an appropriate range increases profits for all parties [17] (this paper utilizes a mathematical model for solving retailers promotion and replenishment decisions under retailer competition and promotional effort with the sales learning curve; an appropriate range for the fraction of promotion cost sharing can achieve channel coordination and determine the distribution of increasable profit). Andrews [18] examines the interplay between two marketing interventions that consumers encounter in retail marketplaces, diagnostic product information, and multiitem sales promotions and discusses that the influence of product information varies as a function of sales promotion format. The effect of sales promotion should be further investigated quantitatively.

Internet sales promotion refers to various activities that stimulate consumer demand, arouse the desire to buy, and facilitate the purchase by using computers and network technology to deliver information about goods and services to the virtual market. With the development of technology, Internet sales promotion has become a major promotion strategy in business management [19]. It plays a positive role in promoting the development of firms and can disseminate promotion information to improve the sales level. Internet sales promotion has many advantages compared to traditional sales promotion. It can push right promotion information to right people, break through the limitation of time and space, and spread around the world around the clock. One successful case of Internet sales promotion is Alibaba's annual global online shopping carnival (OSC) held every November 11 (also known as Double 11 Day or Single's Day). On Singles' Day, this shopping carnival brought the entire Chinese electronic commerce industry an astonishing record volume of transactions worth 91.2 billion RMB in 2015 and 120.7 billion RMB in 2016 [20].

Advertising and sales promotion [21, 22], which are two specific marketing communications tools, account for at least $25 \%$ of UK marketing budgets [23]. From the above literature, Internet sales promotion is rarely considered into a differential game model and studied quantitatively. Motivated by these facts, advertising and Internet sales promotion are introduced into a differential advertising model in this study. This research focuses on the complex dynamics of this model and the effect of Internet sales promotion on sales level. We hope to find a more intuitive way to show how Internet sales promotion affects the market share and optimal sales promotion strategy.

The paper is organized as follows. In Section 2, we introduce the basic model and make this link with practice. The existence and stability of periodic solutions and bifurcation of periodic solutions are investigated in Section 3. In Section 4, different sales promotion strategies are discussed. Numerical results, such as the time series of solutions, bifurcation diagram, and largest Lyapunov exponent, are given in Section 5. Finally, some conclusions are drawn in Section 6.

\section{Model Description}

The following notations will be used in the paper.

$S(t)$ : sales level at time $t$;

$\dot{S}(t)$ : the derivative of $S(t)$ at time $t$;

$u(t)$ : the advertising expenditure at time $t$;

$\Delta S(t)$ : the increment of $S(t)$ at time $t$;

$M$ : the size of the potential market or saturation level;

$T$ : the time between two consecutive Internet sales promotions;

$B$ : the advertising effectiveness of firm;

$H$ : sales promotion target;

$\rho$ : decay constant;

$\lambda$ : the characteristic root;

$r$ : response rate to advertising;

$b$ : a sales promotion parameter reflecting the increment of sales level;

$c$ : the maximum decay constant.

Effective advertising strategy is a response to changes in market conditions and evolves with time. As such, differential models have been generally accepted as a viable approach to the study of advertising decisions. A differential model models the instantaneous variation of market share as a function of the advertising expenditure levels and market 


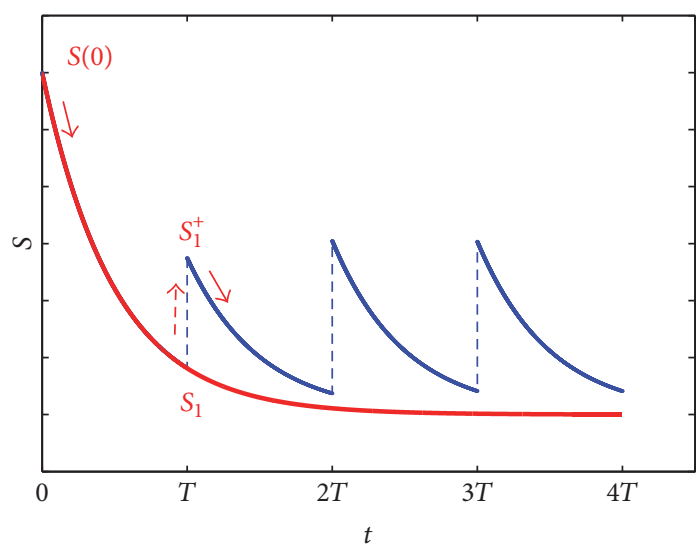

(a)

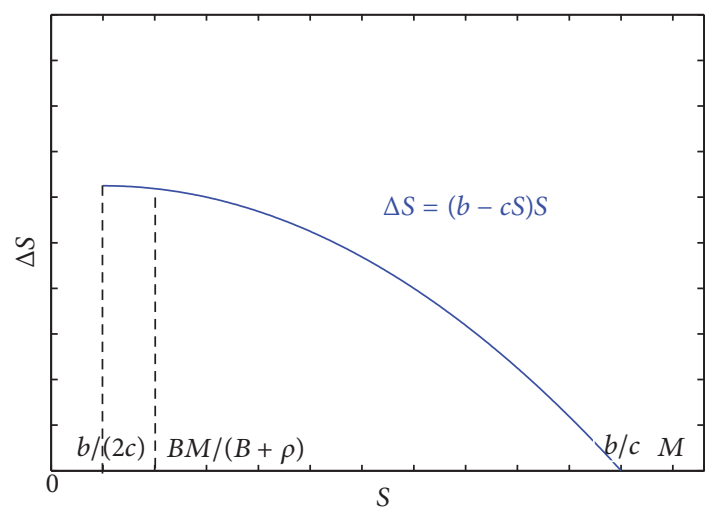

(b)

Figure 1: (a) The solutions of (1) and (4) from the initial point $(0, S(0))$; (b) sketch of Internet sales promotion strategy.

positions. To address the dynamics of advertising decisions, the following advertising model is well studied:

$$
\dot{S}(t)=r u(t)\left(1-\frac{S(t)}{M}\right)-\rho S(t) .
$$

Now consider uniform advertising policy [24], in which the firm advertises at a constant level, in this paper. Hence $u(t)=U, t \geq 0$. Set the advertising effectiveness of firm to be $B=r U / M$ and the initial value $S(0)>B M /(B+\rho)$. One can easily calculate that the solution to system (1) with the initial value $S(0)$ is

$$
S(t)=\left(S(0)-\frac{B M}{B+\rho}\right) \exp [-(B+\rho) t]+\frac{B M}{B+\rho},
$$

$$
t \geq 0 \text {. }
$$

It is observed from Figure 1(a) that the sales level $S(t)$ decreases and tends to $B M /(B+\rho)$.

The five parts of the promotional mix are advertising, sales promotion, personal selling, direct marketing, and public relations. Advertising is the way a company informs potential customers about the products and services it offers in the hope that they will buy eventually, while a sales promotion is an incentive provided to consumers to motivate them to buy immediately. Internet sales promotion [25], including things like online discount promotions, online gift promotions, online lottery promotion, bonus point promotion, and online joint promotion, can stimulate potential customers to action and create an immediate boost in sales volume.

To improve sales, Internet sales promotion strategy is applied here. Suppose that the act of Internet sales promotion is held periodically to create an immediate boost in sales volume and the increment $\Delta S(t)$ depends on sales level $S(t)$, namely,

$$
\Delta S(t)=S\left(t^{+}\right)-S(t)=(b-c S(t)) S(t), \quad t=n T,
$$

where $b>0, c>0, n=1,2, \ldots, S\left(t^{+}\right)=\lim _{\tau \rightarrow 0^{+}} S(t+\tau)$, $b / 2 c \leq B M /(B+\rho)<b / c \leq M$. Figure 1(b) provides a schematic illustration of this Internet sales promotion strategy. The measures to promote sales level are supposed to be taken at moments $t=n T$ and the sales level $S(t)$ turns from $S(n T)$ to $S\left(n T^{+}\right)$, where $S\left(n T^{+}\right)=S(n T)+(b-c S(n T)) S(n T)$ and $(b-c S(n T)) S(n T)$ is the increment of sales.

Now build the following advertising model with Internet sales promotion:

$$
\begin{gathered}
\dot{S}=-\rho S+B(M-S), \quad t \neq n T, \\
S\left(t^{+}\right)=S(t)+(b-c S(t)) S(t), \quad t=n T .
\end{gathered}
$$

System (4) is an impulsive differential system [26]. Figure 1(a) also shows the graph of one solution to (4). The red line shows the decreasing sales level under the influence of decay constant $\rho$ while the blue line shows the change of sales level under Internet sales promotion strategy. The trajectory originating from the initial point $A_{0}(0, S(0))$ reaches the point $A_{1}(T, S(T))$ at $t=T$ and next jumps to the point $A_{1}^{+}\left(T, S\left(T^{+}\right)\right)$due to the effect of sales promotion, and so on. Hence,

$$
\begin{aligned}
S(t)= & \left(S(0)-\frac{B M}{B+\rho}\right) \exp [-(B+\rho) t]+\frac{B M}{B+\rho}, \\
S\left(T^{+}\right)= & S(T)+(b-c S(T)) S(T) . \quad 0 \leq t \leq T . \\
S(t)= & \left(S\left(T^{+}\right)-\frac{B M}{B+\rho}\right) \exp [-(B+\rho)(t-T)] \\
& +\frac{B M}{B+\rho}, \quad T<t \leq 2 T .
\end{aligned}
$$

In the above, Internet sales promotion is introduced into a differential advertising model. In the following paper, complex dynamic properties of system (4) and sales promotion strategy will be investigated analytically. 


\section{Periodic Solutions and Bifurcation}

In order to facilitate management and supply, most firms hope that the market share remains fixed or changes regularly. Therefore, we discuss the periodic change of company's market share under Internet sales promotion strategy in this section.

Suppose the solution of (4) arrives at the point $\left(k T, S_{k}\right)$ at moment $t=k T$; then the solution jumps to point $\left(k T, S_{k}^{+}\right)$ due to the effect of sales promotion and reaches the point $((k+$ 1)T, $\left.S_{k+1}\right)$ at moment $t=(k+1) T$, where $S_{k}=S(k T), S_{k}^{+}=$ $S\left(k T^{+}\right)=S_{k}+\left(b-c S_{k}\right) S_{k}, S_{k+1}=S((k+1) T)$. It follows from (4) that

$$
\begin{aligned}
S((k+1) T)= & \left(S_{k}^{+}-\frac{B M}{B+\rho}\right) \exp [-(B+\rho) T] \\
& +\frac{B M}{B+\rho},
\end{aligned}
$$

and a discrete map

$$
\begin{aligned}
S_{k+1}= & \left(S_{k}+\left(b-c S_{k}\right) S_{k}-\frac{B M}{B+\rho}\right) \exp [-(B+\rho) T] \\
& +\frac{B M}{B+\rho},
\end{aligned}
$$

namely,

$$
S_{k+1}=G_{1} S_{k}^{2}+G_{2}(b) S_{k}+G_{3}:=F\left(S_{k}\right),
$$

where $G_{1}=-c \exp [-(B+\rho) T], G_{2}(b)=(1+b) \exp [-(B+\rho) T]$, $G_{3}=(B M /(B+\rho))(1-\exp [-(B+\rho) T])$.

If $S(k T)=S((k+1) T)$, then the evolution of the dynamics repeats itself. For this to hold, there must be a fixed point $S_{0}$ in discrete map (8); that is,

$$
-G_{1} S_{0}^{2}+\left(1-G_{2}(b)\right) S_{0}-G_{3}=0 .
$$

Note that $-G_{1}>0$ and $G_{3}>0$. Equation (9) has one positive root and one negative root, and the positive root is

$$
S_{01}=\frac{1-G_{2}(b)-\sqrt{\left(G_{2}(b)-1\right)^{2}-4 G_{1} G_{3}}}{2 G_{1}}>0 .
$$

The fixed point $S_{01}$ of map (8) corresponds to the following period- $T$ solution of system (4),

$$
\begin{array}{r}
S(t)=\left(S_{01}+\left(b-c S_{01}\right) S_{01}-\frac{B M}{B+\rho}\right) \\
\cdot \exp [-(B+\rho)(t-n T)]+\frac{B M}{B+\rho}, \\
n T<t \leq(n+1) T,
\end{array}
$$

where $S_{01}$ is shown in (10).

Furthermore, it follows from (8) and (10) that the eigenvalue of the fixed point $S_{0}$ is

$$
\begin{aligned}
\lambda & =F^{\prime}\left(S_{0}\right)=2 G_{1} S_{0}+G_{2}(b) \\
& =1-\sqrt{\left(G_{2}(b)-1\right)^{2}-4 G_{1} G_{3}} .
\end{aligned}
$$

When $|\lambda|<1$, namely,

$$
\begin{aligned}
& -1<1-\sqrt{\left(G_{2}(b)-1\right)^{2}-4 G_{1} G_{3}}<1, \\
& \left(G_{2}(b)-1\right)^{2}-4 G_{1} G_{3}<4,
\end{aligned}
$$

the period-T solution (11) of system (4) is stable. Thus, the discussion above gives the following result.

Proposition 1. Suppose that (13) holds. Then system (4) has a unique stable period-T solution, which is shown in (11).

With the implementation of Internet sales promotion strategy, great changes have taken place in sales level $S(t)$. Proposition 1 shows that sales level $S(t)$ will change periodically with period- $T$ as the sales promotion parameter $b$ meets condition (13). In Proposition 1, the period- $T$ solution is stable under condition $\left(G_{2}(b)-1\right)^{2}-4 G_{1} G_{3}<4$. In the case of $\left(G_{2}(b)-1\right)^{2}-4 G_{1} G_{3}>4$, are there other kinds of periodic solutions in system (4)? To answer this question, it needs to investigate bifurcation of periodic solutions by using the following lemma [27].

Lemma 2. Let $f_{\mu}: \mathbb{R} \rightarrow \mathbb{R}$ be a one-parameter family of map such that $f_{\mu_{0}}$ has a fixed point $x_{0}$ with eigenvalue -1 . Assume the following conditions:

$$
\begin{aligned}
& \left(C_{1}\right)\left((\partial f / \partial \mu)\left(\partial^{2} f / \partial x^{2}\right)+2\left(\partial^{2} f / \partial x \partial \mu\right)\right) \neq 0 \text { at }\left(x_{0}, \mu_{0}\right) ; \\
& \left(C_{2}\right) \bar{a}=(1 / 2)\left(\partial^{2} f / \partial x^{2}\right)^{2}+(1 / 3)\left(\partial^{3} f / \partial x^{3}\right) \neq 0 \text { at }\left(x_{0}, \mu_{0}\right) .
\end{aligned}
$$

Then, there is a smooth curve of fixed points of $f_{\mu}$ passing through $\left(x_{0}, \mu_{0}\right)$, the stability of which changes at $\left(x_{0}, \mu_{0}\right)$. There is also a smooth curve $\gamma$ passing through $\left(x_{0}, \mu_{0}\right)$ so that $\gamma \backslash\left(x_{0}, \mu_{0}\right)$ is a union of hyperbolic period-2 orbits.

In $\left(C_{2}\right)$ the sign of $\bar{a}$ determines the stability and the direction of bifurcation of the orbits of period-2. If $\bar{a}$ is positive, the orbits are stable; if $\bar{a}$ is negative they are unstable.

Suppose the eigenvalue of the fixed point $S_{01}$ is $\lambda=-1$ for $b=b_{0}$; then

$$
\lambda=1-\sqrt{\left(G_{2}\left(b_{0}\right)-1\right)^{2}-4 G_{1} G_{3}}=-1 .
$$

One can calculate that

$$
\begin{aligned}
b_{0} & =\left(1+\sqrt{4+4 G_{1} G_{3}}\right) \exp [(B+\rho) T]-1, \\
S_{01}\left(b_{0}\right) & =\frac{-1-\sqrt{1+G_{1} G_{3}}}{G_{1}} .
\end{aligned}
$$

An eigenvalue with -1 is associated with a flip bifurcation. So $\left(S_{01}\left(b_{0}\right), b_{0}\right)$ is a candidate for flip bifurcation point.

By using (8), it follows that

$$
\begin{aligned}
\frac{\partial F}{\partial b} \frac{\partial^{2} F}{\partial S_{k}^{2}}+2 \frac{\partial^{2} F}{\partial S_{k} \partial b}= & 2 G_{1} \exp [-(B+\rho) T] S_{01} \\
& +2 \exp [-(B+\rho) T]
\end{aligned}
$$




$$
\begin{aligned}
& =-2 \exp [-(B+\rho) T] \sqrt{1+G_{1} G_{3}} \\
& \neq 0 \\
\bar{a} & =\frac{1}{2}\left(\frac{\partial^{2} F}{\partial S_{k}^{2}}\right)^{2}+\frac{1}{3}\left(\frac{\partial^{3} F}{\partial S_{k}^{3}}\right)=2 G_{1}^{2} \\
& >0
\end{aligned}
$$

at $\left(S_{k}, b\right)=\left(S_{01}, b_{0}\right)$.

Conditions $\left(C_{1}\right)$ and $\left(C_{2}\right)$ hold. So a flip bifurcation occurs in view of Lemma 2. A positive period-2T solution bifurcates from the positive period- $T$ solution at $b=$ $b_{0}$. Since $\bar{a}>0$ in $\left(C_{2}\right)$, then the positive period-2T solution is stable. It also means that, for some $\epsilon>0$, system (4) has a positive stable period-2T solution for $b \in$ $\left(b_{0}, b_{0}+\epsilon\right)$. At the same time, the positive period- $T$ solution loses its stability. The analysis above leads to the following results.

Proposition 3. Suppose that $b=b_{0}$, where $b_{0}$ is shown in (15). Then a flip bifurcation occurs at $b=b_{0}$. For some $\epsilon>0$, system (4) has a positive stable period-2T solution for $b \in\left(b_{0}, b_{0}+\epsilon\right)$.

The sales promotion parameter $b$ plays an important role in advertising model. For some $b\left(b<b_{0}\right)$, sales level $S(t)$ changes periodically with period-T. Proposition 3 shows that a flip bifurcation occurs at $b=b_{0}$ and sales level $S(t)$ changes periodically with period-2T for $b \in\left(b_{0}, b_{0}+\epsilon\right)$. Hence system (4) changes from a steady state (period- $T$ ) to another one (period-2T).

To study the period-2T solution in Proposition 3, consider the following iterative map:

$$
\begin{aligned}
S_{k+1}= & F^{2}\left(S_{k}\right) \\
= & G_{1}^{3} S_{k}^{4}+2 G_{1}^{2} G_{2}(b) S_{k}^{3}+\bar{G}_{1}(b) S_{k}^{2}+\bar{G}_{2}(b) S_{k} \\
& +\bar{G}_{3}(b),
\end{aligned}
$$

where $\bar{G}_{1}(b)=G_{1} G_{2}(b)+G_{1} G_{2}^{2}(b)+2 G_{1}^{2} G_{3}, \bar{G}_{2}(b)=G_{2}^{2}(b)+$ $2 G_{1} G_{3} G_{2}(b), \bar{G}_{3}(b)=G_{1} G_{3}^{2}+G_{2}(b) G_{3}+G_{3}$.

Suppose that $S_{0}$ is a fixed point of map (17). Then $S_{0}=$ $F^{2}\left(S_{0}\right)$; namely,

$$
\begin{aligned}
G_{1}^{3} S_{0}^{4} & +2 G_{1}^{2} G_{2}(b) S_{0}^{3}+\bar{G}_{1}(b) S_{0}^{2}+\left(\bar{G}_{2}(b)-1\right) S_{0} \\
& +\bar{G}_{3}(b)=0 .
\end{aligned}
$$

It follows from the facts $S_{01}=F\left(S_{01}\right)$ and $S_{02}=F\left(S_{02}\right)$ that $S_{01}=F^{2}\left(S_{01}\right)$ and $S_{02}=F^{2}\left(S_{02}\right)$, where $S_{01}$ and $S_{02}$ are shown in (10). Hence, $S_{01}$ and $S_{02}$ are also the fixed points of map (17). Furthermore,

$$
\begin{aligned}
& G_{1}^{3} S_{0}^{4}+2 G_{1}^{2} G_{2}(b) S_{0}^{3}+\bar{G}_{1}(b) S_{0}^{2}+\left(\bar{G}_{2}(b)-1\right) S_{0} \\
& \quad+\bar{G}_{3}(b)=\left(S_{0}-S_{01}\right)\left(S_{0}-S_{02}\right)\left[G_{1}^{2} S_{0}^{2}\right. \\
& \left.\quad+\left(G_{1} G_{2}(b)+G_{1}\right) S_{0}+G_{1} G_{3}+G_{2}(b)+1\right] .
\end{aligned}
$$

It follows from (18) that

$$
G_{1}^{2} S_{0}^{2}+\left(G_{1} G_{2}(b)+G_{1}\right) S_{0}+G_{1} G_{3}+G_{2}(b)+1=0 .
$$

Suppose that

$$
\left(G_{2}(b)-1\right)^{2}-4 G_{1} G_{3}>4
$$

holds. A straightforward calculation leads to the two positive roots to (20):

$$
\begin{aligned}
& S_{03}=\frac{-G_{1} G_{2}(b)-G_{1}+\sqrt{\left[G_{1} G_{2}(b)+G_{1}\right]^{2}-4 G_{1}^{2}\left(G_{1} G_{3}+G_{2}(b)+1\right)}}{2 G_{1}^{2}}, \\
& S_{04}=\frac{-G_{1} G_{2}(b)-G_{1}-\sqrt{\left[G_{1} G_{2}(b)+G_{1}\right]^{2}-4 G_{1}^{2}\left(G_{1} G_{3}+G_{2}(b)+1\right)}}{2 G_{1}^{2}} .
\end{aligned}
$$

Thus, $S_{03}$ and $S_{04}$ are fixed points of map (17), $S_{03}=F\left(S_{04}\right)$, and $S_{04}=F\left(S_{03}\right)$.

The positive fixed points $S_{03}$ and $S_{04}$ of map (17) correspond to a period-2T solution of system (4), which starts from the point $\left(0, S_{03}\left(1+b-c S_{03}\right)\right)$, reaches the point $\left(T, S_{04}\right)$, jumps to the point $\left(T, S_{04}\left(1+b-c S_{04}\right)\right)$ due to the effect of sales promotion, and reaches the point $\left(2 T, S_{03}\right)$. The period$2 T$ solution of system (4) is

$$
\begin{aligned}
S(t)= & F_{1}\left(S_{03}\right) \exp [-(B+\rho)(t-n T)]+\frac{B M}{B+\rho}, \\
& n T<t \leq(n+1) T, \\
S(t)= & F_{1}\left(S_{04}\right) \exp [-(B+\rho)(t-(n+1) T)] \\
& +\frac{B M}{B+\rho}, \quad(n+1) T<t \leq(n+2) T,
\end{aligned}
$$

where $F_{1}(x)=(1+b-c x) x-B M /(B+\rho)$. 
Proposition 4. Suppose that (21) holds. Then system (4) has a unique period-2T solution, which is shown in (23).

\section{Sales Promotion Strategy}

Propositions 1 and 3 show that system (4) has stable periodic solutions. The solutions from different initial points will tend to these stable periodic solutions.

Suppose the sales target is that the sales level $S(t)$ is no less than $H$. Now, we discuss the sales promotion strategy to achieve this goal. In the case of stable period- $T$ solution, the sales level $S(t)$ changes according to

$$
\begin{aligned}
& S(t)=\left(S_{01}+\left(b-c S_{01}\right) S_{01}-\frac{B M}{B+\rho}\right) \\
& \cdot \exp [-(B+\rho)(t-n T)]+\frac{B M}{B+\rho}, \\
& n T<t \leq(n+1) T,
\end{aligned}
$$

and $S(t) \in\left[S_{01}, S_{01}+\left(b-c S_{01}\right) S_{01}\right)$.

To achieve the above sales target, it requires that $S_{01} \geq H$. Suppose that $-2 G_{1} H-\left(G_{2}(b)-1\right)>0$; namely,

$$
b \leq\left(1-2 G_{1} H\right) \exp [(B+\rho) T]-1:=\overline{b_{0}} .
$$

It follows from $S_{01} \geq H$ that

$$
\frac{1-G_{2}(b)-\sqrt{\left(G_{2}(b)-1\right)^{2}-4 G_{1} G_{3}}}{2 G_{1}} \geq H,
$$

$$
\begin{aligned}
& \sqrt{\left(G_{2}(b)-1\right)^{2}-4 G_{1} G_{3}} \geq-2 G_{1} H-\left(G_{2}(b)-1\right), \\
& b \geq\left(1-\frac{G_{3}+G_{1} H^{2}}{H}\right) \exp [(B+\rho) T]-1:=b_{1} .
\end{aligned}
$$

If $b_{1}<\min \left\{b_{0}, \overline{b_{0}}\right\}$, the periodic solution (11) is stable for $b_{1} \leq b \leq \min \left\{b_{0}, \overline{b_{0}}\right\}$ in view of Proposition 1 . The analysis above leads to the following results.

Proposition 5. Suppose that $b_{1} \leq b \leq \min \left\{b_{0}, \overline{b_{0}}\right\}$, where $b_{0}$, $\overline{b_{0}}$, and $b_{1}$ are shown in (15), (25), and (26), respectively. Then the sales level $S(t)$ will eventually be no less than $H$ and $S(t) \epsilon$ $\left[S_{01}, S_{01}+\left(b-c S_{01}\right) S_{01}\right)$.

In the case of the period-2T solution, the sales level $S(t)$ changes according to (23).

From (22), it follows that $S_{03}>S_{04}>0$ and $S(t) \epsilon$ $\left[S_{04}, S_{04}+\left(b-c S_{04}\right) S_{04}\right)$. To make sure the sales level $S(t)$ will eventually be no less than $H$ in the case of stable period$2 T$ solution, it requires that $S_{04} \geq H$. Suppose that $-2 G_{1}^{2} H-$ $G_{1} G_{2}(b)-G_{1}>0$; namely,

$$
b \geq\left(-1-2 G_{1} H\right) \exp [(B+\rho) T]-1:=\widetilde{b_{0}} .
$$

It follows from $S_{04} \geq H$ that

$$
\begin{aligned}
\frac{-G_{1} G_{2}(b)-G_{1}-\sqrt{\left[G_{1} G_{2}(b)+G_{1}\right]^{2}-4 G_{1}^{2}\left(G_{1} G_{3}+G_{2}(b)+1\right)}}{2 G_{1}^{2}} & \geq H, \\
\left(1+G_{1} H\right)\left(G_{2}(b)+1\right) & \geq-G_{1} G_{3}-G_{1}^{2} H^{2} .
\end{aligned}
$$

Under condition $1+G_{1} H<0$, one can calculate that

$$
b \leq\left(-1-\frac{G_{1} G_{3}+G_{1}^{2} H^{2}}{1+G_{1} H}\right) \exp [(B+\rho) T]-1:=b_{2} \text {. }
$$

If the period-2T solution (23) is stable for $\max \left\{b_{0}, \widetilde{b}_{0}\right\}<b<$ $b_{2}, S(t)$ will eventually be no less than the threshold $H$.

Proposition 6. Suppose that $1+G_{1} H<0$ and the period$2 T$ solution (23) is stable for $\max \left\{b_{0}, \widetilde{b_{0}}\right\}<b<b_{2}$, where $b_{0}$, $\widetilde{b_{0}}$, and $b_{2}$ are shown in (15), (27), and (29), respectively. Then the sales level $S(t)$ will eventually be no less than $H$ and $S(t) \epsilon$ $\left[S_{04}, S_{04}+\left(b-c S_{04}\right) S_{04}\right)$.

Each firm cares about not only sales, but also profit. For simplicity, stable period-T solution $\left(b<b_{0}\right)$ is used to discuss firm's profit. Set the profit function as

$$
\begin{aligned}
P S(b)= & \int_{0}^{T}\left(\sigma S(t)-u^{2}\right) d t \\
& -p[(b-c S(T)) S(T)]^{2},
\end{aligned}
$$

where $\sigma$ is firm's unit profit margin, $u$ is constant advertising expenditure, $p$ is unit promotion cost, $S(t)$ is shown in (11), $b<b_{0}$, and $S(T)=S_{01}$.

It follows from (11) that

$$
\begin{aligned}
(b & \left.-c S_{01}\right) S_{01} \\
& =\left(S_{01}-\frac{B M}{B+\rho}\right)(\exp ((B+\rho) T)-1),
\end{aligned}
$$

and then

$$
\begin{aligned}
P S(b)= & \int_{0}^{T}\left[\sigma S(t)-u^{2}\right] d t-p\left[\left(b-c S_{01}\right) S_{01}\right]^{2} \\
= & -p\left[\left(S_{01}-\frac{B M}{B+\rho}\right)(\exp ((B+\rho) T)-1)\right]^{2} \\
& +\frac{\exp ((B+\rho) T)-1}{B+\rho} \sigma S_{01}+\frac{\sigma B M T}{B+\rho}-U^{2} T \\
& -\frac{(\exp ((B+\rho) T)-1)^{2} \sigma B M}{(B+\rho)^{2} \exp ((B+\rho) T)} .
\end{aligned}
$$


Hence,

$$
\begin{aligned}
& P S^{\prime}(b) \\
& \quad=\left(-2 p\left(S_{01}-\frac{B M}{B+\rho}\right)(\exp ((B+\rho) T)-1)^{2}\right. \\
& \left.+\frac{\exp ((B+\rho) T)-1}{B+\rho} \sigma\right) S_{01}^{\prime}(b)
\end{aligned}
$$

By using (10), it is easy to see that

$$
\begin{aligned}
& S_{01}^{\prime}(b)=\frac{\exp (-(B+\rho) T)}{2 G_{1}}(1 \\
& \left.-\frac{G_{2}(b)-1}{\sqrt{\left(G_{2}(b)-1\right)^{2}-4 G_{1} G_{3}}}\right) \neq 0, \\
& S_{01}^{\prime \prime}(b)=\frac{2 G_{3} \exp (-2(B+\rho) T)}{\left[\left(G_{2}(b)-1\right)^{2}-4 G_{1} G_{3}\right]^{3 / 2}}>0 .
\end{aligned}
$$

Let $P S^{\prime}\left(b_{3}\right)=0$. Then $b=b_{3}$ is the solution to equation

$$
\begin{gathered}
-2 p\left(S_{01}-\frac{B M}{B+\rho}\right)((\exp ((B+\rho) T)-1))^{2} \\
+\frac{\exp ((B+\rho) T)-1}{B+\rho} \sigma=0 .
\end{gathered}
$$

Similar to the discussion to (26), one can calculate that

$$
b_{3}=\left(1-\frac{G_{3}+G_{1} H_{1}^{2}}{H_{1}}\right) \exp [(B+\rho) T]-1 \text {, }
$$

where $H_{1}=B M /(B+\rho)+\sigma / 2 p(B+\rho)(\exp ((B+\rho) T)-1)$, $\widehat{b_{0}}=b \leq\left(1-2 G_{1} H_{1}\right) \exp [(B+\rho) T]-1$, and $b_{3}<\min \left\{b_{0}, \widehat{b_{0}}\right\}$.

Furthermore,

$$
\begin{aligned}
& P S^{\prime \prime}\left(b_{3}\right) \\
& =\left[-2 p\left(S_{01}-\frac{B M}{B+\rho}\right)(\exp ((B+\rho) T)-1)^{2}\right. \\
& \left.+\frac{\exp ((B+\rho) T)-1}{B+\rho} \sigma\right] S_{01}^{\prime \prime}\left(b_{3}\right) \\
& -2 p[\exp ((B+\rho) T)-1]^{2}\left(S_{01}^{\prime}(b)\right)^{2}=0 \\
& -2 p[\exp ((B+\rho) T)-1]^{2}\left(S_{01}^{\prime}(b)\right)^{2}<0 .
\end{aligned}
$$

In view of the facts $P S^{\prime}\left(b_{3}\right)=0$ and $P S^{\prime \prime}\left(b_{3}\right)<0$, the profit function $P S(b)$ reaches a maximal value at $b=b_{3}$, which gives the following optimal sales promotion strategy.
Proposition 7. Given system (4) and profit function (30). The optimal sales promotion strategy is

$$
\Delta S(t)=\left(b_{3}-c S(t)\right) S(t), \quad t=n T,
$$

where $b_{3}$ is shown in (36).

\section{Numerical Results}

To show the effect of Internet sales promotion strategy and complex dynamics of system (4), numerical results, such as the time series of period- $T$ solution and period-2 $T$ solution, bifurcation diagram, and largest Lyapunov exponents (LLE), are given in this section.

Consider

$$
\begin{gathered}
\dot{S}=-0.9 S+0.1(100-S), \quad t \neq n, \\
S\left(t^{+}\right)=S(t)+(b-0.2 S(t)) S(t), \quad t=n,
\end{gathered}
$$

where $\rho=0.9, B=0.1, M=100, c=0.2$, and $T=1$. One can easily calculate that $B M /(B+\rho)=10, G_{1} \approx-0.0736$, $G_{2}(b)=\exp (-1)(1+b)$, and $G_{3} \approx 6.3212$.

Set $b=4.8$ in system (39). The positive fixed point of map (15) is $S_{01} \approx 19.7571$, and hence $S_{01}^{+} \approx 36.52$. Figure 2(a) shows that system (39) has a period- $T$ solution, which starts from the initial point $(0,36.52)$ and reaches $(1,19.7571)$ at $t=1$. Since $\left(G_{2}(b)-1\right)^{2}-4 G_{1} G_{3} \approx 3.1456<4$, condition (13) holds. Figure 2(b) shows that the solution starting from the point $(0,50)$ tends to the period- $T$ solution in Figure 2(a). Illustrative simulations shown in Figure 2 are in agreement with Proposition 1.

A straightforward calculation gives $b_{0}=(1+$ $\left.\sqrt{4+4 G_{1} G_{3}}\right) \exp [(B+\rho) T]-1 \approx 5.6945$. It follows from Proposition 3 that flip period-doubling bifurcation occurs at $b=5.6945$. One-dimensional bifurcation diagram and largest Lyapunov exponents (LLE) in Figure 3(a) can well show the dynamic characteristics of system (39) with the sales promotion parameter $b$ changes. Along with the increase of $b$ from 5 to 5.6945 , system (39) has a stable period- $T$ solution and hence is in stable state. System (39) is in stable state with period-2T when $b$ changes from 5.6945 to 7.25 while system is in stable state with period-4T when $b$ changes from 7.25 to 7.55 . When the sales promotion parameter $b>$ 7.85 , system (39) will go into chaotic state. The LLE in Figure 3(a) corresponding to the bifurcation diagram can also show the system stability features. The LLE equals zero for $b=5.6945$, and flip bifurcation occurs. When the first time LLE > 0 , the system goes into chaotic state. Simulations shown in Figure 3(a) verify Proposition 3. In a word, the sales promotion parameter $b$ can cause the advertising model to demonstrate complicated character.

Set $b=5.9$ in (39). Then $\left(G_{2}(b)-1\right)^{2}-4 G_{1} G_{3} \approx 4.3993>$ 4 , and condition (21) holds. One can calculate that $S_{03} \approx$ 27.2830, $S_{04} \approx 20.8084, S_{04}^{+}=S_{04}+\left(5.9-0.2 S_{04}\right) S_{04} \approx 56.98$, $S_{03}^{+}=S_{03}+\left(5.9-0.2 S_{03}\right) S_{03} \approx 39.38$. Figure 3(b) shows the unique period- $2 T$ solution, which starts from $(0,56.98)$, reaches the point $(1,27.283)$, jumps to the point $(1,39.38)$ due to the effect of sales promotion, arrives at the point $(2,20.8084)$, jumps to the point $(2,56.98)$ due to the effect of 


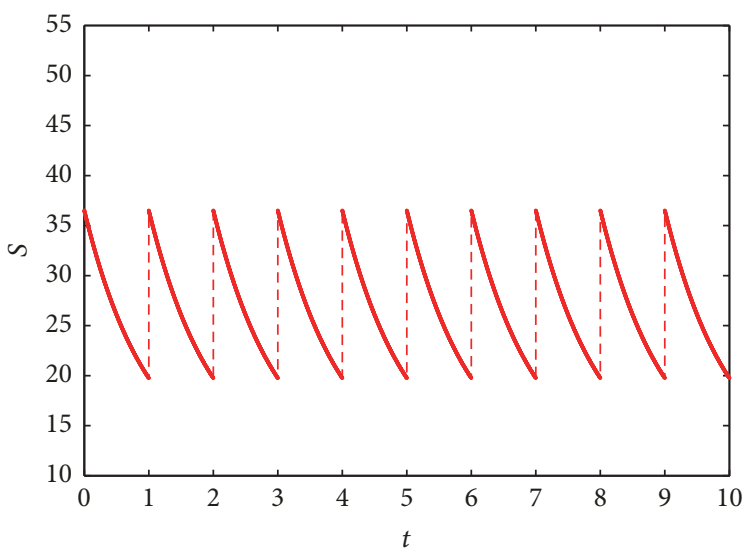

$--S(0)=36.52$

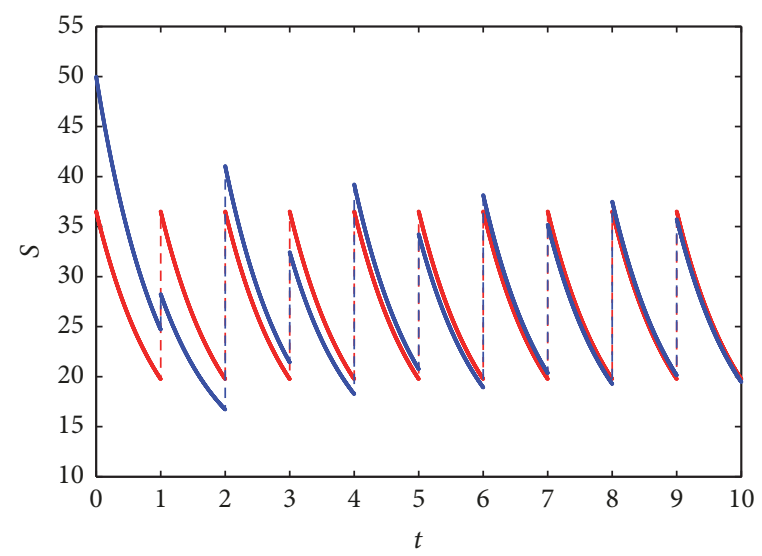

$--S(0)=36.52$

-. $S(0)=50$

(a)

(b)

FIgURE 2: (a) The period- $T$ solution to (39) with $b=4.8$ and the initial point $(0,36.52)$; (b) two solutions to system (39) with the initial points $(0,36.52)$ and $(0,50)$.

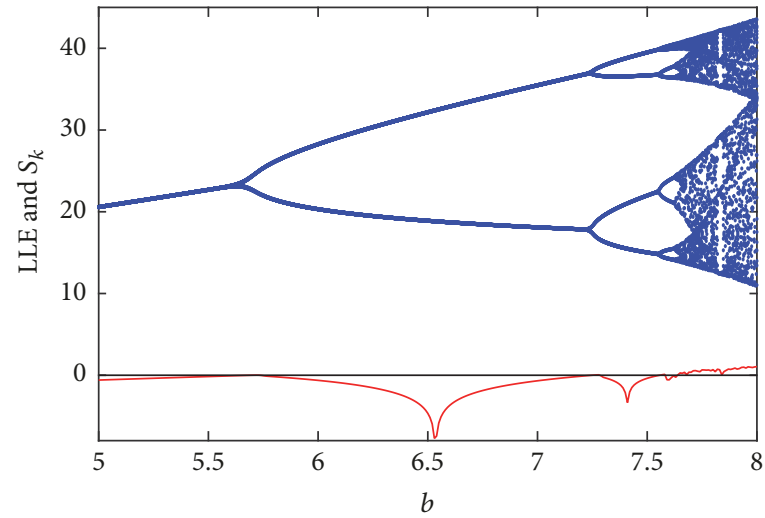

(a)

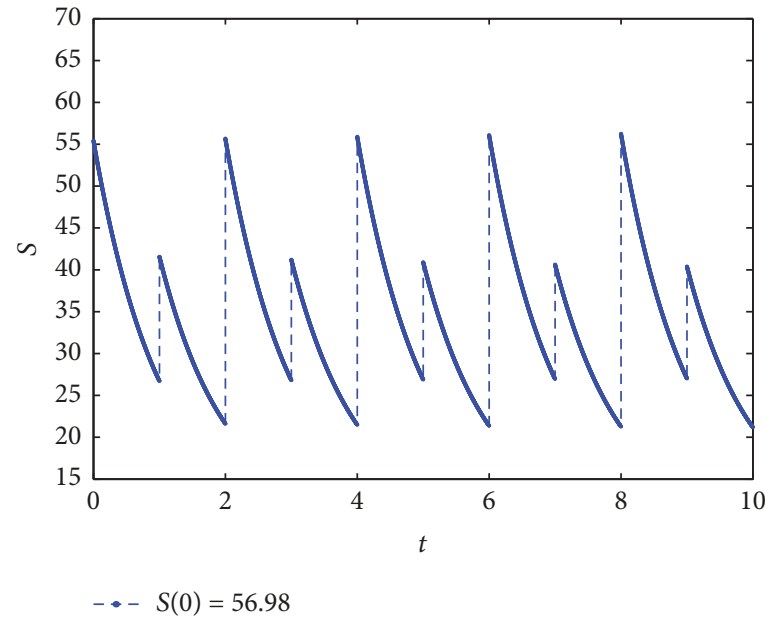

(b)

Figure 3: (a) The bifurcation diagram and largest Lyapunov exponent of system (39) for $b \in(5,8)$; (b) the period-2T solution of (39) with $b=5.9$ and the initial point $(0,56.98)$.

sales promotion, and so on. Simulation shown in Figure 3(b) is in agreement with Proposition 4.

Figure 3(a) also shows that the path to chaos is perioddouble bifurcation. The period-4T solution to system (39) with $b=7.25$ is shown in Figure 4(a) while the solution to system (39) with $b=7.85$ is shown in Figure 4(b).

The value of $b_{1}(25)$ for $H \in(10,23.5)$ is shown in Figure 5(a). Choose $H=20$; it follows that $b_{1} \approx 4.859$. Now set $b=5$ in system (39). It is observed from Figure 5(b) that the value of $S(t)$ will be eventually larger than $H=20$, which is in agreement with Proposition 5.

Figure 6(a) shows the value of $b_{2}$ (27) for $H \in(18,23)$. Choose $H=20$; it follows that $b_{2} \approx 6.084$. Now set $b=6$ in system (39). Conditions $1+G_{1} H \approx-0.472<0$ and $b_{0}<b<$ $b_{2}$ hold. It is observed from Figure 6(b) that the value of $S(t)$ will be eventually larger than $H=20$, which is in agreement with Proposition 6.

Set the profit function as

$$
\begin{aligned}
P S(b)= & \int_{0}^{T}(20 S(t)-200) d t \\
& -p\left[\left(b-0.2 S_{2}(T)\right) S_{2}(T)\right]^{2} .
\end{aligned}
$$

Take $p=0.6$. A straightforward calculation gives that $b_{4} \approx$ 4.756 and the optional sales promotion strategy is

$$
\Delta S(t)=(4.756-0.2 S(t)) S(t), \quad t=n .
$$

Figure 7(a) shows the value of the profit PS (40) for $p=$ 0.6 and $b \in(2,5.7)$ while Figure 7(b) shows the value of 


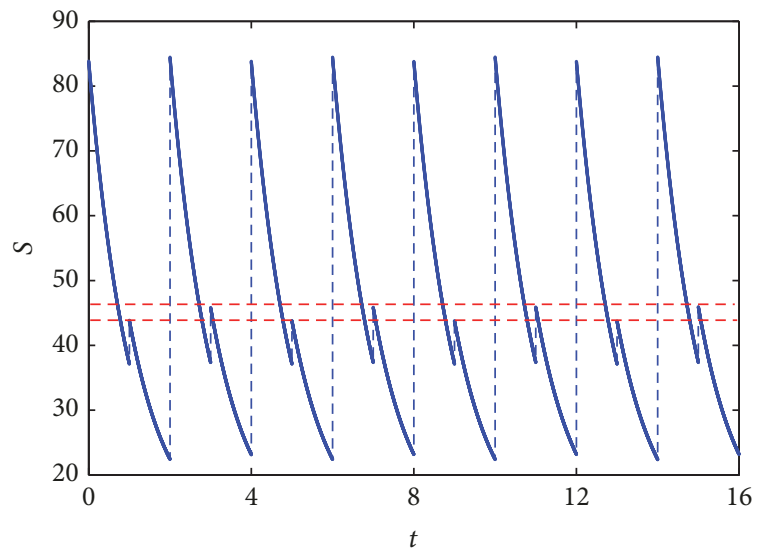

(a)

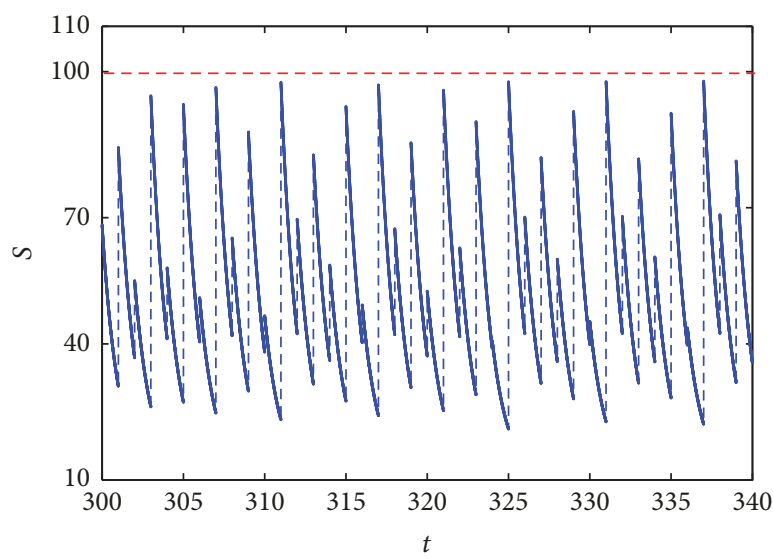

(b)

Figure 4: (a) The period-4T solution of (39) with $b=7.25$; (b) the solution of (39) with $b=7.85$.

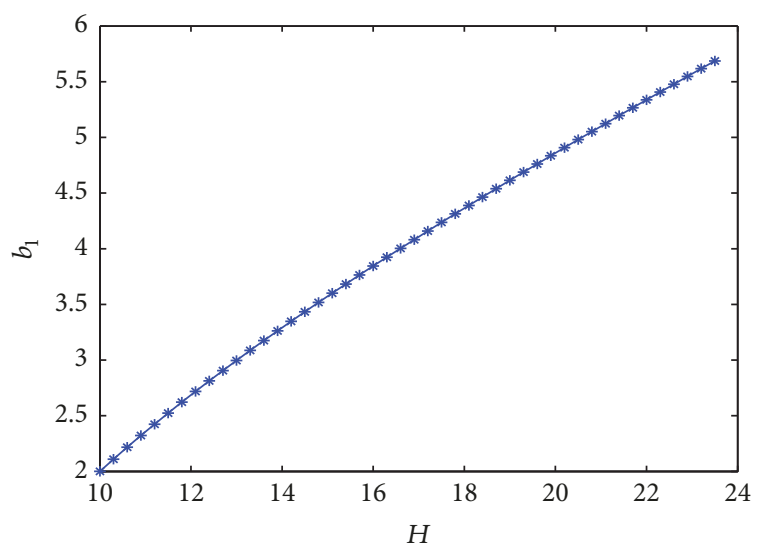

(a)

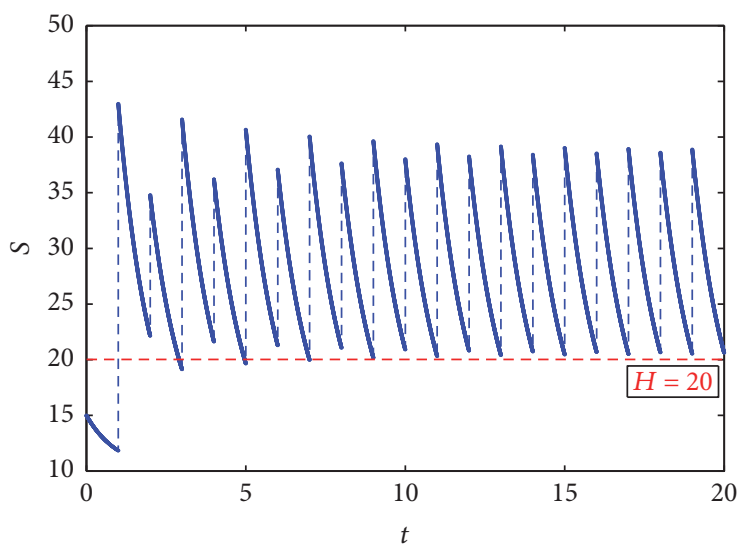

(b)

FIgure 5: (a) The value of $b_{1}$ for $H \in(10,23.5)$; (b) the solution of (39) with $b=5$ and the initial point $(0,15)$.

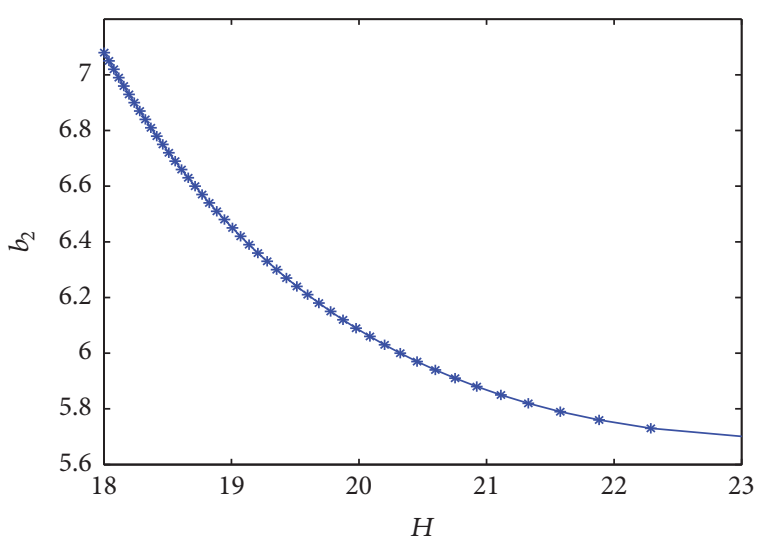

(a)

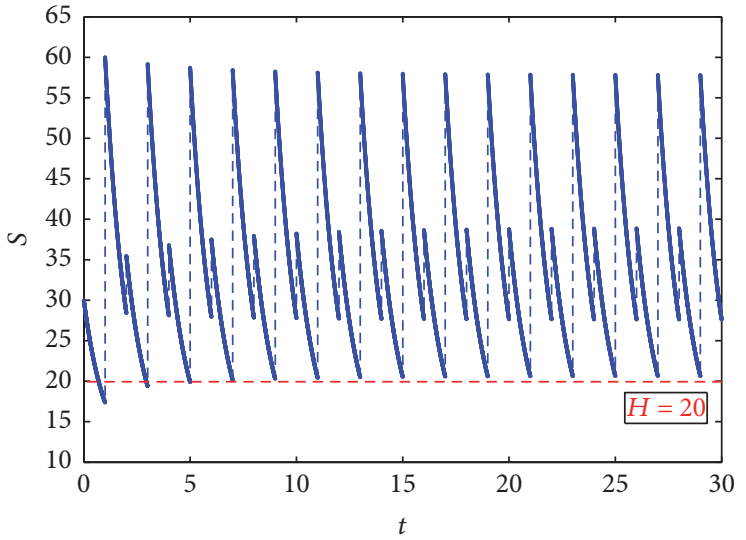

(b)

Figure 6: (a) The value of $b_{2}$ for $H \in(18,23)$; (b) the solution of (39) with $b=6$ and the initial point $(0,30)$. 


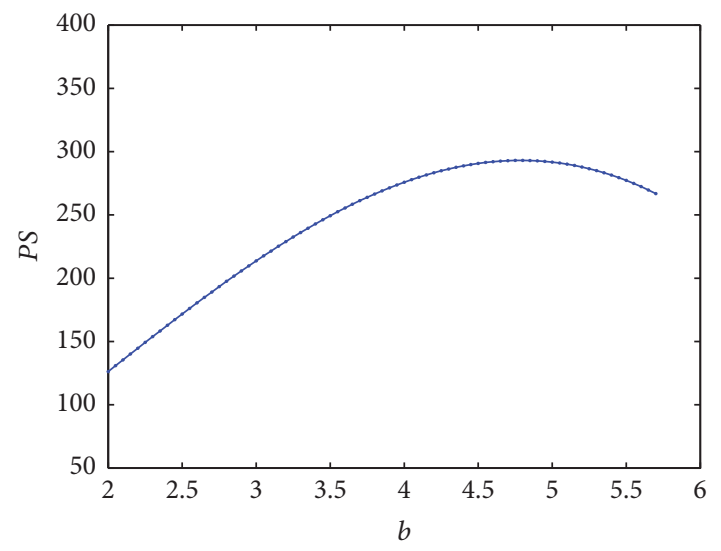

(a)

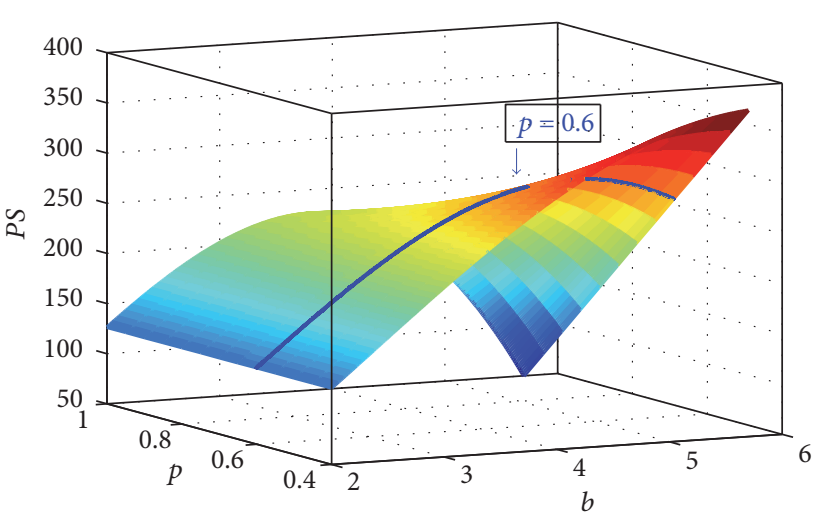

(b)

Figure 7: (a) The value of $P S(b)(40)$ for $p=0.6$ and $b \in(2,5.7)$; (b) the value of $P S(b)(40)$ for $p \in(0.4,1)$ and $b \in(2,5.7)$.

the profit $P S(40)$ for $p \in(0.4,1)$ and $b \in(2,5.7)$. These numerical results are in agreement with Proposition 7.

\section{Conclusions}

In this paper, Internet sales promotion is introduced into a differential advertising model. Results show that the system possesses complex dynamic behavior. Advertising model has a unique stable period- $T$ solution or period-2 $T$ solution for parameter $b$ satisfies some conditions. In these cases of periodic solutions, sales level $S(t)$ tends to a periodic solution and eventually changes periodically, which provides regulation to direct manufacture. The sales promotion parameter $b$, which can modify the system stability and lead to chaos through flip bifurcation, plays an important role in the sales promotion strategy.

Internet sales promotion strategy is proposed to improve sales level and maximize firm's profit. Suppose the sales target is that the sales level $S(t)$ is no less than $H$. The firm should choose the sales promotion parameter $b$ such that conditions in Propositions 5 and 6 hold. Profit is a key element in firm's decision to employ what sales promotion strategy. The optimal sales promotion strategy is obtained and used to maximize firm's profit in Proposition 7. These results have some significant theoretical and practical values in related markets.

\section{Conflicts of Interest}

The authors declare that there are no conflicts of interest regarding the publication of this paper.

\section{Acknowledgments}

This work is jointly supported by the Natural Science Foundation of China under no. 11662001 and the Science Foundation of Guangxi Province under no. 2016GXNSFDA380031.

\section{References}

[1] N. Fukawa and Y. Zhang, "Profit-sharing between an opensource firm and application developers - maximizing profits from applications and in-application advertisements," Industrial Marketing Management, vol. 48, pp. 111-120, 2015.

[2] C. Zhang and H. Zheng, "D3-Equivariant coupled advertising oscillators model," Procedia Eng, vol. 192, pp. 342-347, 2011.

[3] C. F. Nicholson and H. M. Kaiser, "Dynamic market impacts of generic dairy advertising," Journal of Business Research, vol. 61, no. 11, pp. 1125-1135, 2008.

[4] A. Buratto, L. Grosset, and B. Viscolani, "Advertising channel selection in a segmented market," Automatica, vol. 42, no. 8, pp. 1343-1347, 2006.

[5] G.-I. Bischi, L. Stefanini, and L. Gardini, "Synchronization, intermittency and critical curves in a duopoly game," Mathematics and Computers in Simulation, vol. 44, no. 6, pp. 559-585, 1998.

[6] G. I. Bischi and M. Kopel, "Multistability and path dependence in a dynamic brand competition model," Chaos, Solitons \& Fractals, vol. 18, no. 3, pp. 561-576, 2003.

[7] F. Wu and J. Ma, “The complex dynamics of a multi-product mixed duopoly model with partial privatization and crossownership," Nonlinear Dynamics, vol. 80, no. 3, pp. 1391-1401, 2015.

[8] J. Ma and Z. Guo, "The parameter basin and complex of dynamic game with estimation and two-stage consideration," Applied Mathematics and Computation, vol. 248, pp. 131-142, 2014.

[9] J. Huang, M. Leng, and L. Liang, "Recent developments in dynamic advertising research," European Journal of Operational Research, vol. 220, no. 3, pp. 591-609, 2012.

[10] X. Su, H. Liu, and S. Hou, "The Trilateral Evolutionary Game of Agri-Food Quality in FARmer-Supermarket Direct Purchase: A SIMulation APProach," Complexity, Article ID 5185497, 11 pages, 2018.

[11] F. M. Feinberg, "On continuous-time optimal advertising under S-shaped response," Management Science, vol. 47, no. 11, pp. 1476-1487, 2001.

[12] A. Prasad and S. P. Sethi, "Integrated marketing communications in markets with uncertainty and competition," Automatica, vol. 45, no. 3, pp. 601-610, 2009.

[13] A. Krishnamoorthy, A. Prasad, and S. P. Sethi, "Optimal pricing and advertising in a durable-good duopoly," European Journal of Operational Research, vol. 200, no. 2, pp. 486-497, 2010. 
[14] X. Su, S. Duan, S. Guo, and H. Liu, "Evolutionary Games in the Agricultural Product Quality and Safety Information SYStem: A MUltiagent SIMulation APProach," Complexity, Article ID 7684185, 13 pages, 2018.

[15] M. Palazon and E. Delgado-Ballester, "The expected benefit as determinant of deal-prone consumers response to sales promotions," Journal of Retailing and Consumer Services, vol. 18, no. 6, pp. 542-547, 2011.

[16] D. DelVecchio, D. H. Henard, and T. H. Freling, "The effect of sales promotion on post-promotion brand preference: A metaanalysis," Journal of Retailing, vol. 82, no. 3, pp. 203-213, 2006.

[17] Y. Tsao and G. Sheen, "Effects of promotion cost sharing policy with the sales learning curve on supply chain coordination," Computers \& Operations Research, vol. 39, no. 8, pp. 1872-1878, 2012.

[18] D. Andrews, "Product information and consumer choice confidence in multi-item sales promotions," Journal of Retailing and Consumer Services, vol. 28, pp. 45-53, 2016.

[19] H. Martin, K. Eva, and M. Radovan, The importance of e-mail marketing in e-commerce. Marketing Communication: Modern Promotional Strategy, Ronald Press Company, New York, NY, USA, 2017.

[20] X. Xu, Q. Li, L. Peng et al., "The impact of informational incentives and social influence on consumer behavior during Alibabas online shopping carnival," Comput Hum Behav, vol. 76, pp. 245-254, 2017.

[21] C. W. Park, M. S. Roth, and P. F. Jacques, "Evaluating the effects of advertising and sales promotion campaigns," Industrial Marketing Management, vol. 17, no. 2, pp. 129-140, 1988.

[22] I. Buil, L. de Chernatony, and E. Martínez, "Examining the role of advertising and sales promotions in brand equity creation," Journal of Business Research, vol. 66, no. 1, pp. 115-122, 2013.

[23] J. Byun and S. Jang, "Effective promotions for membership subscriptions and renewals totourist attractions: Discount vs. bonus," Tourism Management, vol. 50, pp. 194-203, 2015.

[24] H. I. Mesak, A. Bari, and Q. Lian, "Pulsation in a competitive model of advertising-firm's cost interaction," European Journal of Operational Research, vol. 246, no. 3, pp. 916-926, 2015.

[25] H. K. Cheng and K. Dogan, "Customer-centric marketing with Internet coupons," Decision Support Systems, vol. 44, no. 3, pp. 606-620, 2008.

[26] D. D. Bainov and P. S. Simeonov, "Impulsive Differential Equations: Periodic Solutions and Applications," Tech. Rep., Longman Scientific \& Technical, New York, NY, USA, 1993.

[27] J. Guckenheimer and P. Holmes, Nonlinear Oscillations, Dynamical Systems, and Bifurcations of Vector Fields, Springer-Verlag, New York, NY, USA, 1983. 


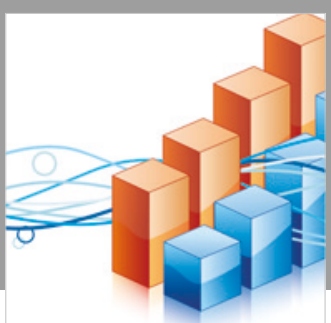

Advances in

Operations Research

\section{-n-m}
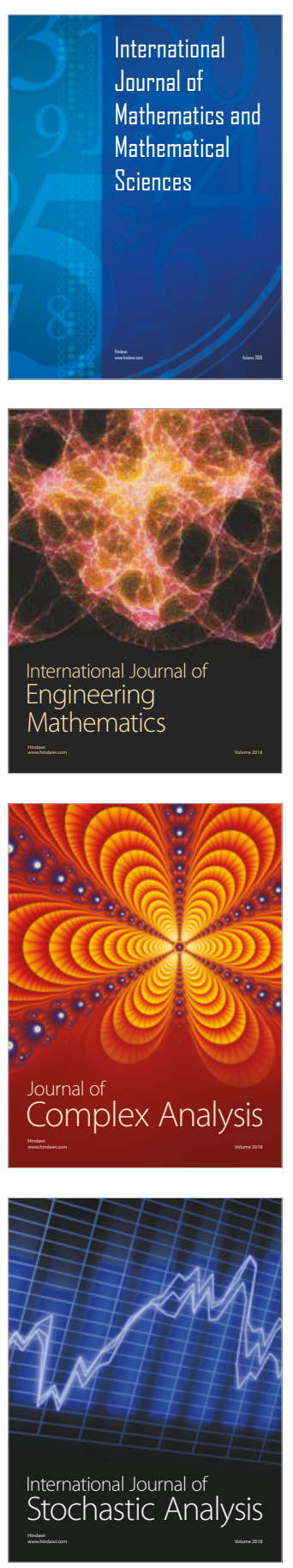
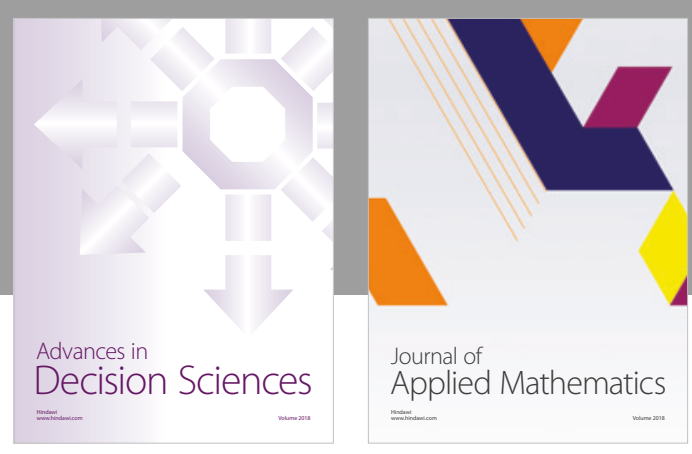

Journal of

Applied Mathematics
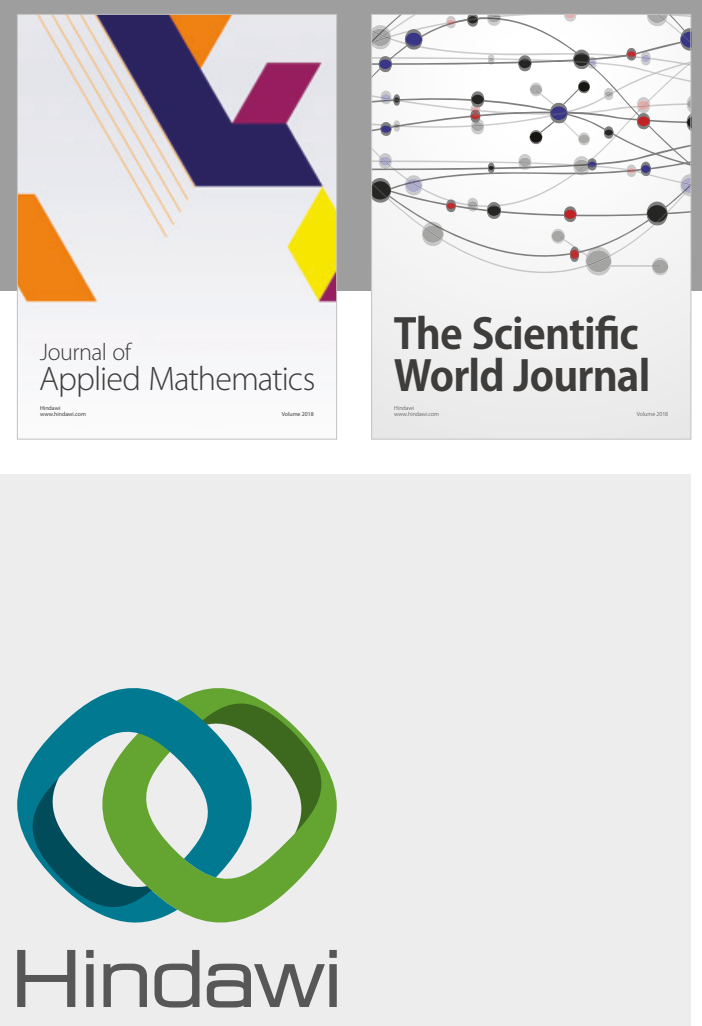

Submit your manuscripts at

www.hindawi.com

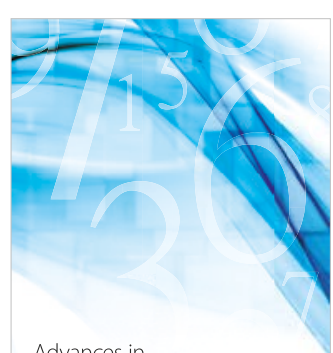

Advances in
Numerical Analysis
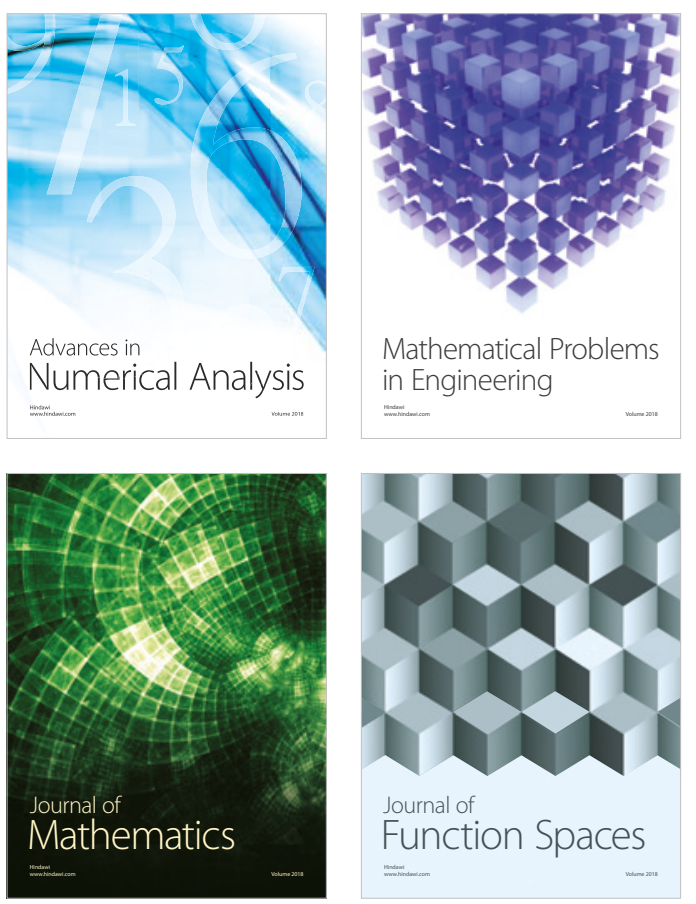

Mathematical Problems in Engineering

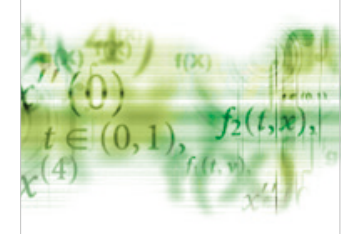

International Journal of

Differential Equations

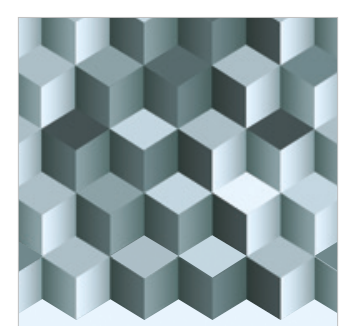

Journal of

Function Spaces

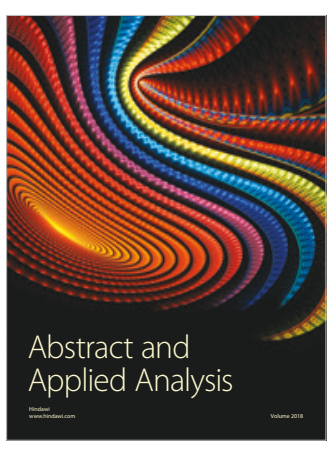

The Scientific

World Journal

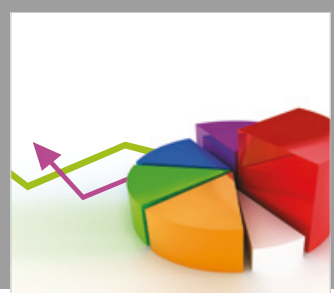

Journal of

Probability and Statistics
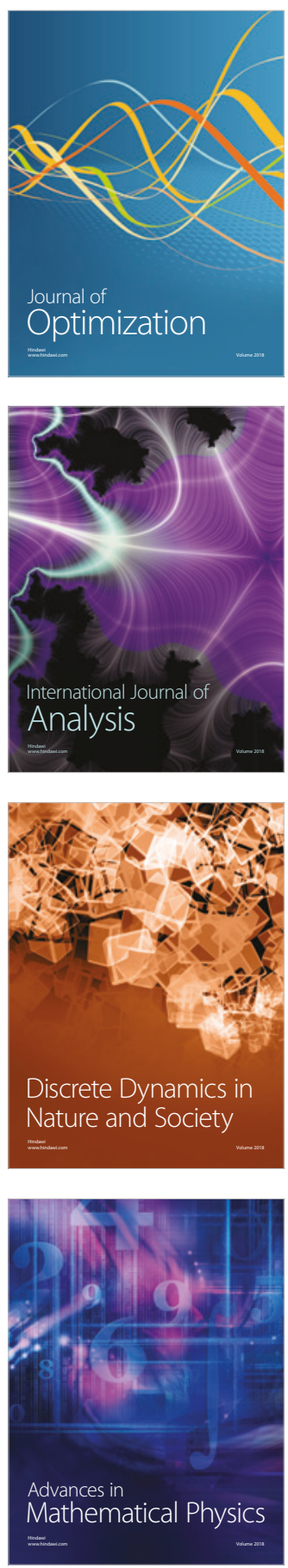